\title{
Near-field measurements versus far-field estimations of air gun array sound pressure levels and sound exposure levels for blended source acquisition.
}

Philip Fontana* and Marc Rocke, Polarcus

Copyright 2019, SBGf - Sociedade Brasileira de Geofísica

This paper was prepared for presentation during the $16^{\text {th }}$ International Congress of the Brazilian Geophysical Society held in Rio de Janeiro, Brazil, 19-22 August 2019.

Contents of this paper were reviewed by the Technical Committee of the $16^{\text {th }}$ International Congress of the Brazilian Geophysical Society and do not necessarily represent any position of the SBGf, its officers or members. Electronic reproduction or storage of any part of this paper for commercial purposes without the written consent of the Brazilian Geophysical Society is prohibited.

\begin{abstract}
Calibrated near-field hydrophone (NFH) measurements from individual air gun array elements and full production arrays are used to demonstrate that far-field signature computer models of source sound pressure levels (SPL) are significantly greater than the actual levels introduced into the environment local to the air gun sources.
\end{abstract}

Analysis of the NFH measurements has also been extended to evaluate SPL and sound exposure levels (SEL) expected from various blended source acquisition techniques.

\section{Introduction}

During the past 20 years or so there has been steadily increasing concerns about potential impacts from sound levels emitted by seismic survey air gun arrays on marine fauna, cetaceans in particular.

These concerns have spurred increasing activity in research efforts to characterize the sound fields generated by air gun arrays and the propagation of the emitted acoustic energy in the marine environment local to a seismic survey.

In parallel, many government agencies responsible for granting seismic survey permits have included acoustic monitoring and mitigation procedures as part of the permit requirements. Most permit applications require a description of the seismic source and an estimate of the expected acoustic output from the source. Typically, the acoustic source levels submitted with the applications are derived from computer model estimates of the Sound Pressure Levels (SPL) generated in the far-field of a specific air gun array design. These levels are then often used as input to compute monitoring and mitigation parameters.

This approach has an inherent set of problems in that the estimated far-field SPLs are then extrapolated back to a virtual receiver point $1 \mathrm{~m}$ from a virtual point source. The key word is virtual and the problem is that the quoted acoustic levels for the virtual point source never actually occur in the environment. The reason is that an air gun array, by design, has a spatial distribution of elements that do not behave as a point source emitter at $1 \mathrm{~m}$ from the geometric center of the array. Therefore, the point source estimate only becomes valid at some distance away from the center of the array where the arrivals from each of the individual array elements add together coherently and appear as if the total energy is emanating from a single point source emitter at a distance. In other words, that is the definition of the far-field of an array. In general terms the far-field distance can be computed as:

$$
d=\left(f * a^{2}\right) / c
$$

Where $f$ is frequency in $\mathrm{Hz}$; $a$ is the greatest spatial dimension of the array in meters; $c$ is the speed of sound in water. (Richardson et al, 1995).

Distances of $d$ and greater are referred to as the far-field of the array. Distances less than $d$ are referred to as the near-field of the array. It is interesting to note the dependence of the far-field distance on emitted frequency. The higher the frequency, the greater the distance from near-field to far-field.

We propose that for purposes of acoustic mitigation requirements, maximum near-field acoustic levels be used to determine maximum expected sound pressure levels in the vicinity of a seismic air gun array and not a level extrapolated from a far-field estimate of a virtual point source emitter. Measurements in the near-field of the array will show significantly lower acoustic levels than what are normally predicted from virtual point source estimations.

\section{Method}

In practice, most seismic air gun arrays are equipped with an array of near-field hydrophones, usually one for each array element (Figure 1). For an example 2-string array (Figure 2) there will be $12 \mathrm{NFH}$ per array.

In a triple source production configuration three sets of the 2-string array will be deployed providing an array of $36 \mathrm{NFHs}$ in the immediate vicinity of the full seismic source spread (Figure 3)

Prior to the start of a survey a NFH recording of each individual air gun in the array is recorded for $\mathrm{QC}$ purposes. From these single air gun and cluster element recordings the zero-to-peak (0-P) SPL for each air gun and cluster can be measured at each NFH location in the array, in isolation from interference from the other array elements. 
Figure 4 shows the calibrated $\mathrm{NFH}$ recordings of a single $45 \mathrm{in}^{3}$ air gun as recorded on the local NFH (Channel 1) and a remote NFH (Channel 13) approximately at a distance, $\boldsymbol{r}$, about $25 \mathrm{~m}$ away.

Figure 5 shows the location of calibrated NFHs and center of source locations for three consecutive shot sequences of triple source acquisition. The NFH recordings for these shots were analyzed for maximum 0 $P$ amplitude for all 36 NFHs per shot. These values were then plotted against the distance each hydrophone was from the active source center. Figure 6 shows the results of these measurement in bar-m (6a) and dB re $1 \mathrm{uPa}-\mathrm{m}$ (6b) for all NFHs for all three shots.

\section{Discussion}

In figure $6 \mathrm{~b}$ the maximum amplitude recorded at any $\mathrm{NFH}$ in the spread for the three example production shots is $244.5 \mathrm{~dB}$ (16.9 bars). This value compares to a 0-P estimate of about $262 \mathrm{~dB}$ produced by a popular air gun signature modelling program. There is a significant departure between the far-field predicted virtual point source response versus the actual maximum SPLs emitted by a working air gun array. The actual maximum SPL is only about $13 \%$ of the value that would be entered into a permit application based upon the modeling output.

There is one issue that requires clarification in this comparison, the bandwidth of the signals used to produce the values. The $262 \mathrm{~dB}$ value from the modelling program is for a bandwidth of $25 \mathrm{kHz}$ while the bandwidth of the $\mathrm{NFH}$ measurements is $800 \mathrm{~Hz}$. Figure 7 shows the first 1 $\mathrm{kHz}$ band of the spectrum, in red, of an air gun array farfield signature measured out to $25 \mathrm{kHz}$. There are a number of interesting features in this plot, but the most germane to this discussion is that a very high percentage of the acoustic energy in the air gun signature is produced at frequencies below about $300 \mathrm{~Hz}$. Therefore, there should not be a significant difference in comparing measurements recorded to $800 \mathrm{~Hz}$ vs those recorded to much higher frequencies.

This plot also highlights a significant problem in using computer models to estimate the high frequency emissions of air gun arrays, the models produce significantly higher values at high frequencies than the measurement. Also, as mentioned above, the near-field to far-field transition distance is a function of frequency. The 2-string array from which the NFH data were record has a maximum spatial dimension of $14 \mathrm{~m}$. That means the far field extrapolation for a $300 \mathrm{~Hz}$ bandwidth signal is valid from about 39 from the array center while that distance is about $390 \mathrm{~m}$ for a $3 \mathrm{kHz}$ bandwidth signal.

These observations also bring up some interesting implications for blended seismic source acquisition. It appears that the maximum SPLs from any source configuration are generated by the largest elements in the array design and occur in the near-field of the array. So, the actual maximum SPLs emitted into the local environment may not be much different whether firing simultaneous source arrays, overlapping shot source arrays, or encoded sequencing of individual elements in an array. During the presentation we will show results where we used the measured $\mathrm{NFH}$ signatures to model the implications of total SPL and estimated SEL using various blended source designs.

\section{Conclusions}

Instead of using a single SPL value derived for a virtual point source in the permit process, the frequency and near-field vs far-field aspects of an air gun array design should also be taken into account when designating pragmatic monitoring and mitigation zones. The SPL values actually emitted into the local environment are significantly lower than predicted by far-field signature simulations.

\section{Acknowledgments}

We thank the crew of Polarcus Naila for the time and effort to ensure accurate calibration of the NFHs.

\section{References}

Richardson, W.J., Green, C.R., Malme, C.I., and Thomson, D.H., 1995

Marine Mammals and Noise

Academic Press, San Diego

Arslan M. Tashmukhambetov, Natalia A. Sidorovskaia, George E. Ioup, Juliette W. Ioup, Joal J. Newcomb, Christopher D. Walker, Ben Brock, and Grayson H. Raybor, 2006, 3-D airgun source characterization and propagation modeling, SEG Technical Program Expanded Abstracts 2015: pp. 100-104 


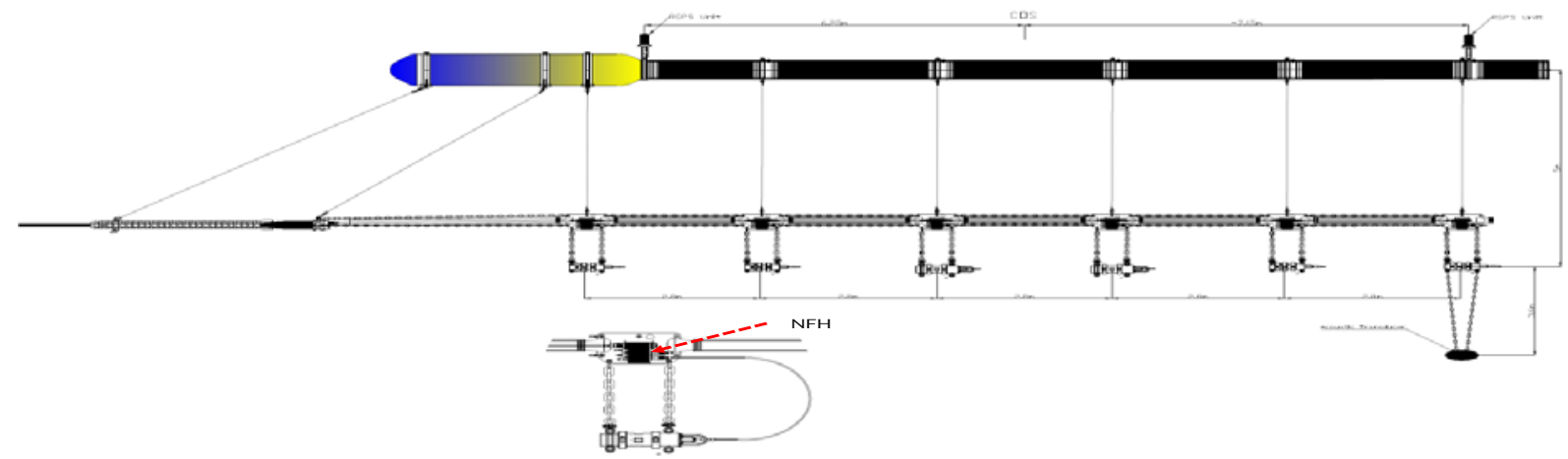

Figure 1: Position of Near-Field Hydrophone (NFH) relative to air gun

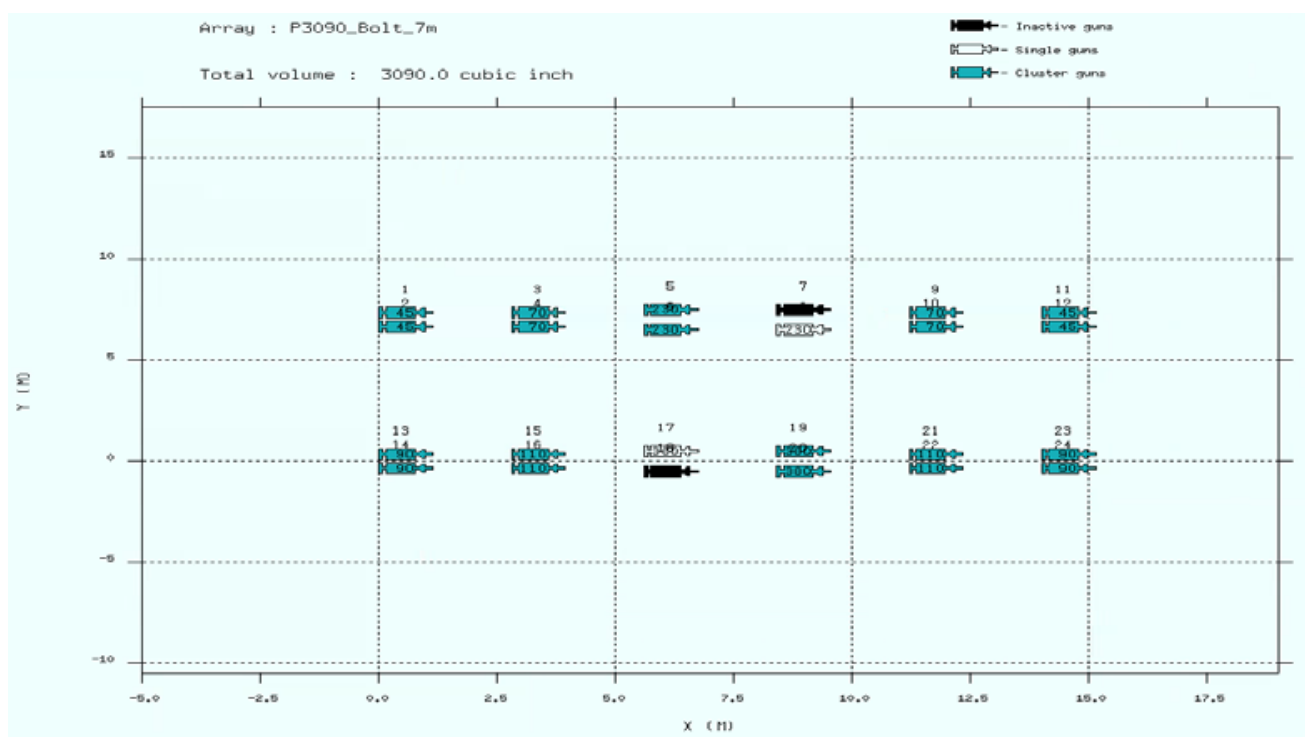

Figure 2: 2-string air gun array with twelve active elements and one NFH per element

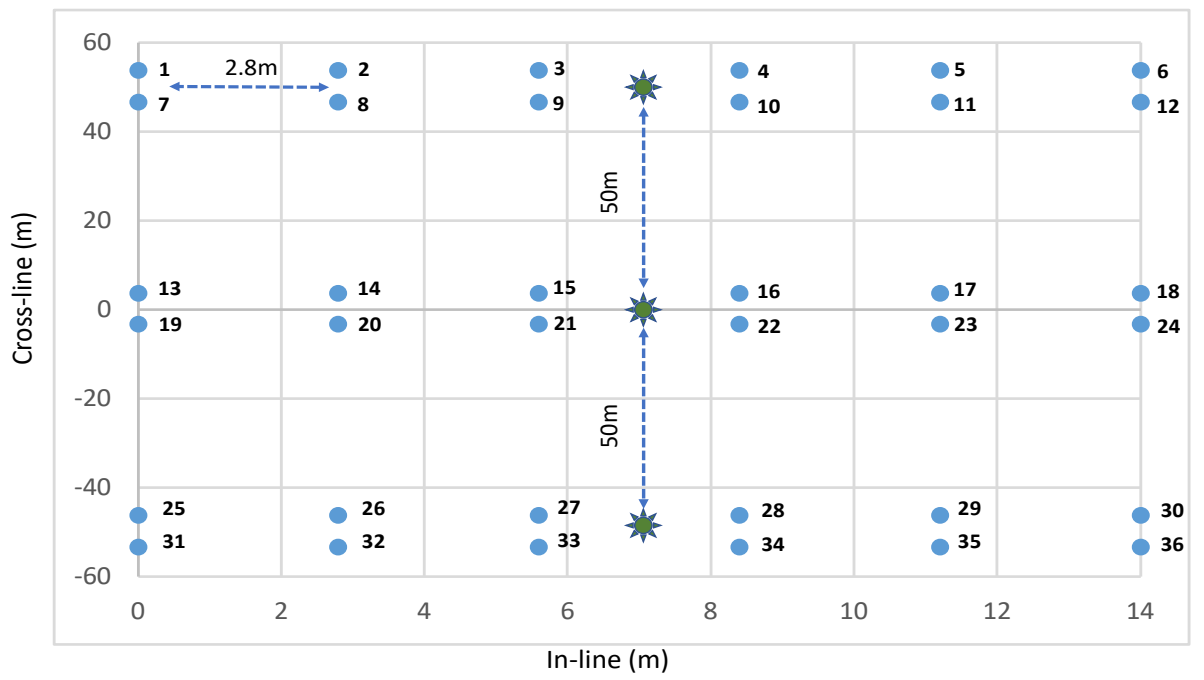

Figure 3. Lay out of NHFs on triple 2-string source arrays with NFH recording channels numbered. 


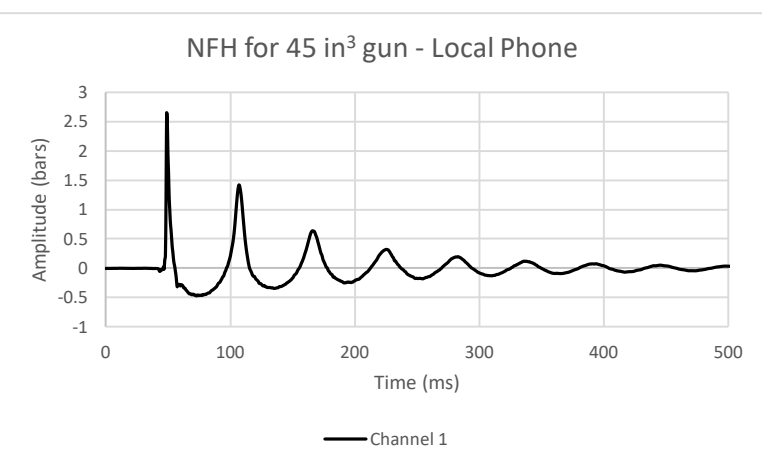

0-P Amplitude $=2.65$ bars or $228.5 \mathrm{~dB}$ re $1 \mathrm{uPa}$

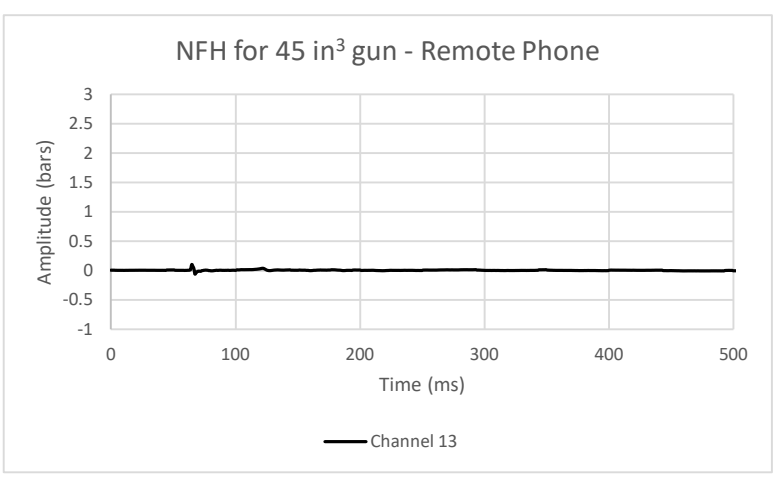

0-P Amplitude $=0.1$ bars or $200 \mathrm{~dB}$ re $1 \mathrm{uPa}$

Figure 4. Time signatures of a single $45 \mathrm{in}^{3}$ air gun as measured by the local NFH and a NFH about 25m away.

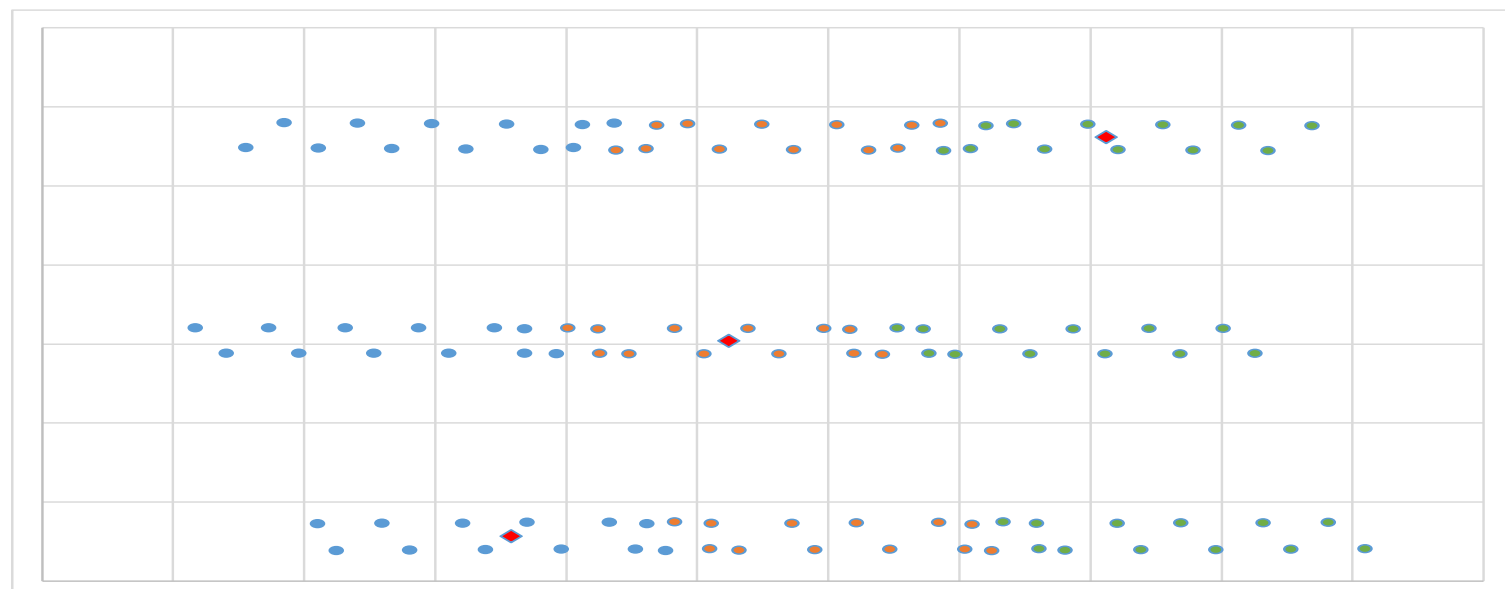

Figure 5: Location of source centers (stars) and NFHs (ovals) for three consecutive shots. Blue = shot 1, Red = shot 2, and Green $=$ shot 3
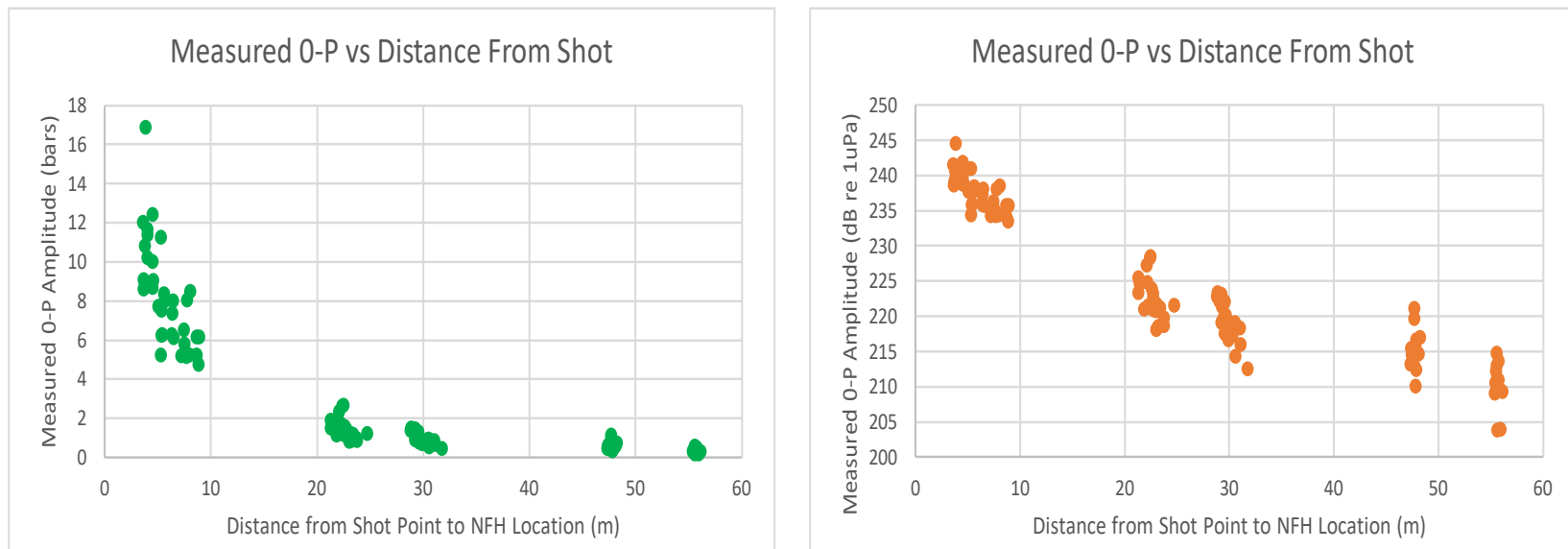

Figure 6: NFH measured 0-P amplitude vs distance from center of shots 


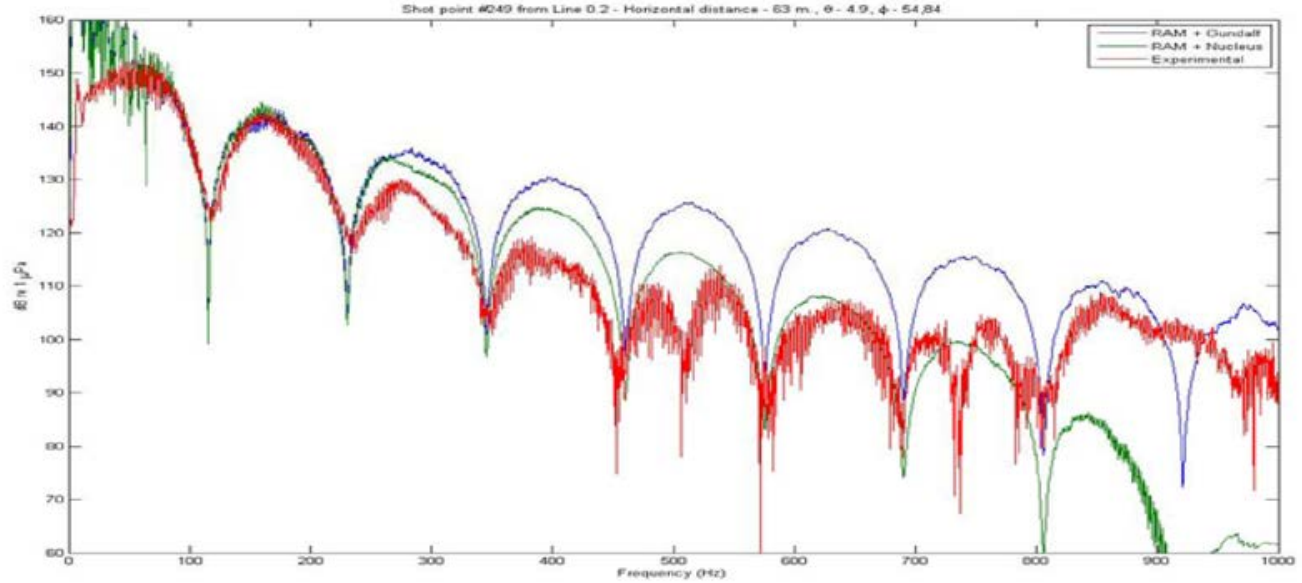

Fig.3: Comparison of measured and simulated seismic signals for shot 249.

Figure 7. Computed vs measured high bandwidth air gun signatures (Tashmukambetov A.M, et al 2006) 